\title{
Efficient Placement of an Aerial Relay Drone for Throughput Maximization
}

\author{
Hazim Shakhatreh $\mathbb{D}^{1},{ }^{1}$ Ali Alenezi $\mathbb{D}^{2,3}$ Ahmad Sawalmeh $\mathbb{D}^{2,3}{ }^{3,4}$ Muhannad Almutiry $\mathbb{D}^{2,3}$ \\ and Waed Malkawi iD ${ }^{1}$ \\ ${ }^{1}$ Department of Telecommunications Engineering, Hijjawi Faculty for Engineering Technology, Yarmouk University, Irbid, Jordan \\ ${ }^{2}$ Electrical Engineering Department, Northern Border University, Arar, Saudi Arabia \\ ${ }^{3}$ Remote Sensing Unit, Northern Border University, Arar, Saudi Arabia \\ ${ }^{4}$ Computer Science Department, Northern Border University, Arar, Saudi Arabia
}

Correspondence should be addressed to Hazim Shakhatreh; hazim.s@yu.edu.jo

Received 21 February 2021; Revised 1 April 2021; Accepted 20 May 2021; Published 8 June 2021

Academic Editor: Tran Manh Hoang

Copyright (c) 2021 Hazim Shakhatreh et al. This is an open access article distributed under the Creative Commons Attribution License, which permits unrestricted use, distribution, and reproduction in any medium, provided the original work is properly cited.

\begin{abstract}
Unmanned aerial vehicle (UAV) communication can be used in overcrowded areas and either during or postdisaster situations as an evolving technology to provide ubiquitous connections for wireless devices due to its flexibility, mobility, and good condition of the line of sight channels. In this paper, a single UAV is used as an aerial relay node to provide connectivity to wireless devices because of the considerable distance between wireless devices and the ground base station. Specifically, two path loss models have been utilized; a cellular-to-UAV path loss for a backhaul connection and an air-to-ground path loss model for a downlink connection scenario. Then, the tradeoff introduced by these models is discussed. The problem of efficient placement of an aerial relay node is formulated as an optimization problem, where the objective is to maximize the total throughput of wireless devices. To find an appropriate location for a relay aerial node that maximizes the overall throughput, we first use the particle swarm optimization algorithm to find the drone location; then, we use three different approaches, namely, (1) the equal power allocation approach, (2) water filling approach, and (3) modified water filling approach to maximize the total users' throughput. The results show that the modified water filling outperforms the other two approaches in terms of the average sum rate of all users and the total number of served users. More specifically, in the best-case scenario, it was observed that the average sum rate of the modified water filling is better than the equal power allocation and ensuring $100 \%$ coverage. In contrast, the water filling provides a very close average sum rate to the modified water filling, but it only provides a $28 \%$ user coverage.
\end{abstract}

\section{Introduction}

While most UAV research works in wireless communication demonstrate UAV uses as an aerial base station [1-5], a recent growing trend is to use UAV as a relay node [6-12]. One classification for the UAVs deployment as relay nodes is conducted based on the objective function, as shown in Figure 1.

UAV can be deployed as a relay node when the infrastructure of the terrestrial base station is damaged due to natural disasters or overloaded during crowded events [13-15]. Specifically, UAV relay can be used to provide wireless coverage between ground wireless devices and the remote terres- trial base station without reliable direct communication links [16-19].

On the one hand, many works utilize UAV as a relay node in wireless communication. For example, the study in [6] proposed to use a UAV as a relay node to minimize the UAV transmission power. Moreover, in [7], the authors presented an energy-efficient deployment of a relay UAV such that the latency of backhaul link capacity and the delay in the backhaul link are minimized. Furthermore, Kumar et al. in [8] proposed to use a UAV as a relay node such that the quality of service requirement was guaranteed. In addition to that, in [9], the authors developed a closed-form to find the optimum placement of the UAV acts as a relay node 


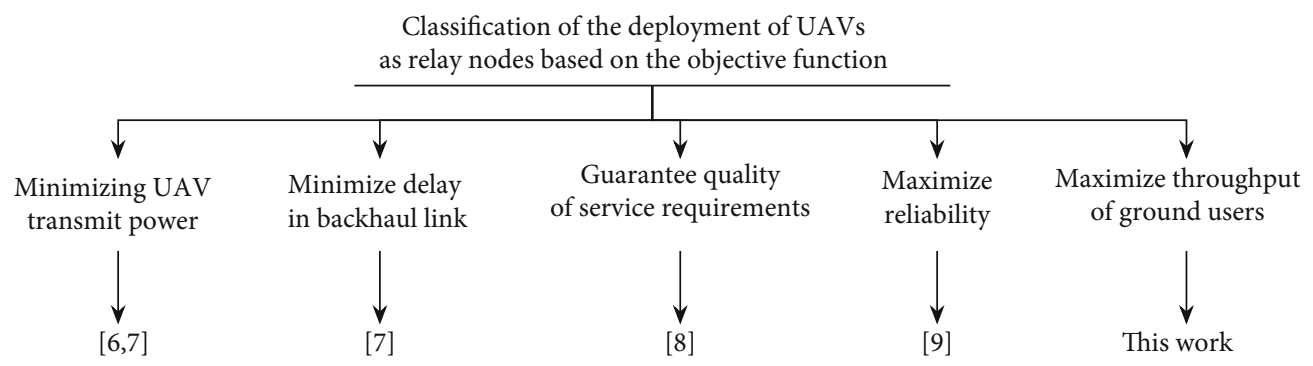

FIgURE 1: Classification of the deployment of UAVs as relay node based on the objective function.

where the objective is to maximize the reliability of the network.

On the other hand, Zhan et al. in [10] proposed a multiUAVs relaying system between ground devices and a remote base station. A fixed-wing UAV relay node was used to provide a connection between ground devices and the base station in a single hop. They utilized a free space path loss with line-of-sight connections between devices and the relay node. They focused on the physical layer communication, link throughput, the symbol error rate, and handoff algorithm for ground devices to switch between relay nodes.

Moreover, in [11], a relay selection problem was investigated for UAV-assisted vehicular ad hoc networks (VANETs). They study the cooperation between multiUAVs, the network node mobility model, and the air-toground link quality of service for UAV relay selection in VANETs. While in our work, we propose an efficient placement of a stationary UAV to act as a relay node between a remote base station and ground users. Unlike the works in $[10,11]$, we propose using a stationary UAV acts as a relay node along with practical path losses models presented in $[1,20]$ to provide wireless connectivity between ground devices and a remote base station where the objective is to maximize the throughput of ground devices.

Inspired by the aforementioned studies on relay's UAV network, this work proposed to use a single UAV acts as a relay node between a remote terrestrial base station and the ground users, where the objective is to find the efficient placement of the UAV such that the overall throughput of the network is maximized. The cellular-to-UAV path loss model presented in [20] and the air-to-ground path loss model presented in [1] have been employed for the backhaul connection and the downlink connection scenarios, respectively. To the best of our knowledge, this is the first work that proposes to use a drone as a relay node to optimize the wireless device's throughput using practical path loss models presented in $[1,20]$.

We summarize the main contributions of this work as follows:

(i) We utilize a cellular-to-UAV path loss model for the backhaul link [20] and an air-to-ground path loss model for the downlink [1,21]; then, we discuss the tradeoff introduced by these models

(ii) We formulate the problem of efficient placement of a single UAV, where the objective is to maximize ground users' total throughput (iii) We propose to use the particle swarm optimization algorithm [22, 23] and the water-filling algorithm [24] to search for the optimal placement for a drone that maximizes the throughput and demonstrates the effectiveness of the proposed algorithm under different cases

The rest of this paper is structured as follows. Section 2 presents the related works. Then, in Section 3, the system model is discussed. In Section 4, we formulate the optimization problem, where the objective is to maximize the total throughput of ground users. In Section 5, we propose to use the particle swarm optimization algorithm and the waterfilling algorithm to search for optimal placement for the aerial relay node. In Section 6, we present the simulation results and analysis. Finally, Section 7 presents the conclusion and future work.

\section{Related Works}

In the literature, many researchers consider the deployment strategies of UAVs as relay nodes by optimizing different objective functions such as (1) minimizing its transmit power and maximizing energy efficiency $[3,6,7],(2)$ minimizing the delay in backhaul and delay aware UAV positioning [7], and (3) guaranteeing the quality of service (QoS) requirements [8]. Figure 1 presents the classification of the deployment of UAVs as relay nodes based on the objective function.

The authors in [20] developed a statistical behavior path loss model between a cellular base station and a flying UAV. The value of the path loss was reported as a function of the depression angle and the cellular coverage beneath the UAV. They proposed a cellular to UAV communication channel capable of capturing the mean path loss between a UAV and the serving terrestrial base station. This model was derived based on comprehensive experimental data measurements for both terrestrial and aerial coverage in a typical suburban area. The model offers a reliable and simple cellular to UAV path loss prediction that can be useful for researchers and network operators.

Many researchers utilized the path loss model in [20] to use UAV as a relay node. The authors in [6] proposed an energy-efficient approach using a UAV that acts as a relay node on a cellular network to serve all ground users inside the coverage area. They investigated an uplink resource allocation that involves the power and time allocation of the relay UAV node for scheduling the uplink transmission of the 
users to optimize the uplink sum rate of the UAV rely-aided cellular network.

The study in [7] proposed an energy-efficient deployment strategy of UAV acts as flying relay roadside unit, that considering the latency of backhaul link capacity and the vehicular users (VUs) constraints such that the total power consumption was minimized. More specifically, they investigated a 3D deployment approach of a $\mathrm{UAV}$ as a flying relay roadside unit to minimize the total power consumption, where the latency in the UAV network and backhaul link problem was considered. In this work, the energy-efficiency, latency, and backhaul problems were combined into one optimization problem. First, the latency constraint for the UAV network and backhaul link capacity was derived as a function of the distance between the UAV and the terrestrial base station. Then, they proposed a communication problem to obtain the optimal location of the aerial relay node and the power allocation vector of vehicular users.

In [8], the authors utilized a UAV-enabled base station (UBS) to provide wireless coverage to the region where terrestrial infrastructure network goes out of service due to natural disaster, malfunction, or crowded events. They proposed cellular broadband access using UBS, which guaranteed the quality of service requirement.

In [9], UAV's optimum placement as a relay for maximum reliability is investigated. The maximum reliability performance measures they considered are the total power loss, the overall outage, and the overall bit error rate (BER), which are derived and optimized numerically for both decode-and-forward (DF) and amplify-and-forward (AF) scenarios. The optimum placement is studied for both static and mobile UAVs. The numerical results show that the performance measures have slightly different optimum altitudes and that the DF has better performance than AF.

In [25], the authors derived a closed-form outage probability function for the UAV that serves as a relay between the ground base station and multiple users in 3D. The communication channel between the BS and the UAV is assumed to follow the Rician channel model. In contrast, the channel between the UAV and the end-users is assumed to follow the Rayleigh fading model. Due to the derived function's complexity, they used the asymptotic outage probability function that agrees with the closed-form function at high SNRs to optimize the UAV's 3D location. The numerical results show that the increase in UAV transmits power more than the BS transmit power does not improve the system's performance.

Works from previous studies use a drone as a relay node under the assumption of free space propagation to increase the throughput of wireless devices. This condition may not be realistic for urban environments in particular. Also, they study the optimal 3D placement of an aerial relay node that can enhance multiple objective functions. In this paper, we plan to use realistic path loss models for an aerial relay node to improve the throughput of wireless devices where the data rates between the ground base station and users are low due to the considerable distance.

\section{System Model}

3.1. System Settings. Let $\left(X_{u}, Y_{u}, Z_{u}\right)$ denote the placement of the aerial relay node. We consider that $M$ wireless devices are located far away from the base station. These wireless devices cannot communicate with the remote base station due to the limited transmitting capacity. The wireless devices must be served by the drone that acts as an aerial relay node to send the information from the ground base station to ground users, as shown in Figure 2. We assume a downlink scenario in which the UAV uses a frequency division multiple access (FDMA) technique to send information to the wireless devices at a signal-to-noise ratio (SNR) greater than or equal $\mathrm{SNR}_{\mathrm{th}}$. FDMA assigns each user one subchannel for communication, so the links will not interfere with each other. We also consider that the aerial base station's maximum transmit power is $p^{\max }$.

3.2. Path Loss Models. Most of the air-ground channel measurements focus on large-scale statistics such as path loss exponent and shadow fading [26]. The path loss model between the ground base station and the aerial relay node is given as [20]:

$$
\begin{aligned}
L_{\mathrm{BS}-\mathrm{UAV}}\left(d_{2 D}, \theta\right)= & 10 \alpha \log \left(d_{2 D}\right)+A\left(\theta-\theta_{o}\right) \exp \left(-\frac{\theta-\theta_{o}}{B}\right) \\
& +\eta_{o}+\mathcal{N}\left(0, a \theta+\sigma_{o}\right)
\end{aligned}
$$

where $d_{2 D}=\sqrt{\left(X_{u}-X_{\mathrm{BS}}\right)^{2}+\left(Y_{u}-Y_{\mathrm{BS}}\right)^{2}}$ is the 2D distance between the projection of the UAV and the base station, $\theta$ the depression angle, $\alpha$ is the terrestrial path loss exponent, $A$ excess path loss scaler, $\theta_{o}$ is angle offset, $B$ is angle scaler, $\eta_{o}$ excess path loss offset, $\mathscr{N}\left(0, a \theta+\sigma_{o}\right)$ is a Gaussian random variable with $a$ is UAV shadowing slope, and $\sigma_{o}$ is UAV shadowing offset.

The path loss model between the aerial relay node and a ground user $i \in M$ is given as $[1,21]$ :

$$
L_{\mathrm{UAV}-i}\left(d_{3 D}, \phi\right)=P(\mathrm{LOS}) \cdot L_{\mathrm{LOS}}+P(\mathrm{NLOS}) \cdot L_{\mathrm{NLOS}},
$$

where $d_{3 D}=\sqrt{\left(X_{u}-X_{i}\right)^{2}+\left(Y_{u}-Y_{i}\right)^{2}+\left(Z_{u}\right)^{2}}$ is the 3D distance between the UAV and a ground user, $P(\mathrm{LOS})$ is the probability of having line of sight (LOS) connection at an evaluation angle of $\phi, P(\mathrm{NLOS})$ is the probability of having non-LOS connection and equal (1-P(LOS)), and $L_{\mathrm{LOS}}$ and $L_{\mathrm{NLOS}}$ are the average path loss for LOS and NLOS paths.

3.3. Path Loss Model Tradeoff. Note that there is a critical trade-off in the path loss models when the horizontal distance between the aerial relay node and the wireless device changes. When this distance increases, the path loss between the drone and ground wireless device (i.e., $L_{\mathrm{UAV}-i}$ ) increases, while the path loss between the ground base station and the drone (i.e., $L_{\mathrm{BS}-\mathrm{UAV}}$ ) decreases. Similarly, when this horizontal distance decreases, the path loss between the drone and ground wireless device (i.e., $L_{\mathrm{UAV}-i}$ ) decreases, while the path 


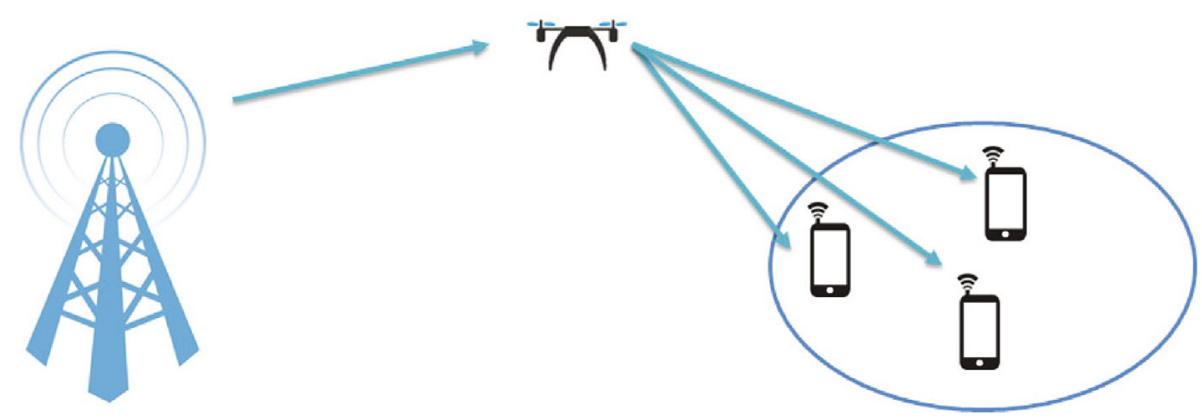

Figure 2: System model.

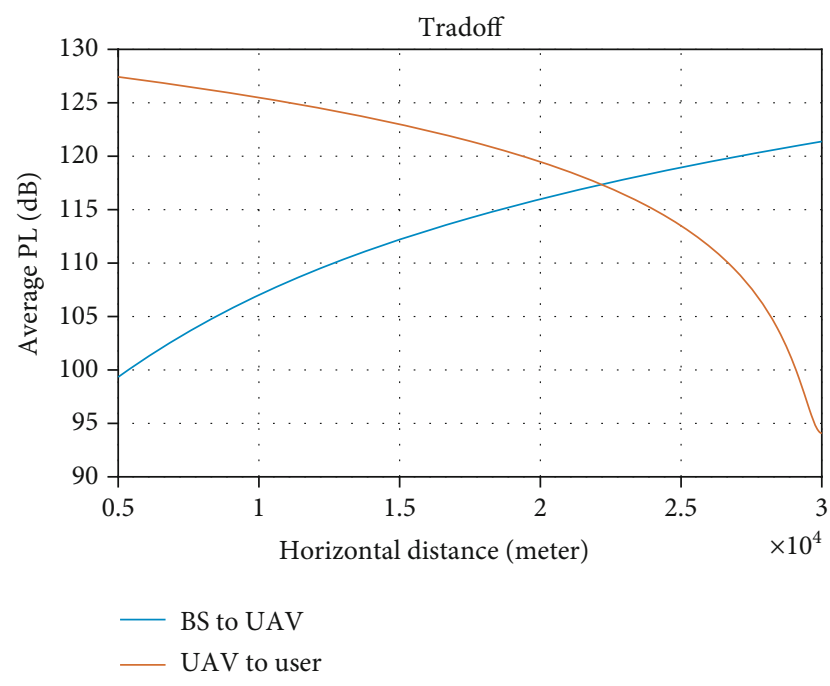

FIGURE 3: Path loss model tradeoff.

loss between the ground base station and the drone (i.e., $L_{\mathrm{BS}-\mathrm{UAV}}$ ) increases (see Figure 3 ).

\section{Problem Formulation}

Consider a wireless communication between the ground base station and a ground user via the aerial relay node. The data rate of the link that connected the ground base station with the aerial relay node is given by:

$$
C_{\mathrm{BS}-\mathrm{UAV}}=B_{\mathrm{BS}} \cdot \log _{2}\left(1+\mathrm{SNR}_{\mathrm{BS}-\mathrm{UAV}}\right) \text {, }
$$

where $B_{\mathrm{BS}}$ is the transmission bandwidth of the ground base station, and $\mathrm{SNR}_{\mathrm{BS}-\mathrm{UAV}}$ is the signal-to-noise ratio at the aerial relay node. The data rate of the link that connected the aerial relay node with a ground user is given by:

$$
C_{\mathrm{UAV}-i}=B_{\mathrm{UAV}-i} \cdot \log _{2}\left(1+\mathrm{SNR}_{\mathrm{UAV}-i}\right) \text {, }
$$

where $B_{\mathrm{UAV}-i}$ is the transmission bandwidth of the aerial relay node, and $\mathrm{SNR}_{\mathrm{UAV}-i}$ is the signal-to-noise ratio at a ground user. Let us assume that each wireless device has a channel with bandwidth equals $B_{\mathrm{UAV}} / M$, where $B_{\mathrm{UAV}}$ is the aerial relay node bandwidth, and $M$ is the number of wireless devices. Then, the power needed to reach the data rate $R_{i}$ for each wireless device is given by:

$$
p_{i}=\left(2^{R_{i} \cdot M / B_{U A V}}-1\right) \cdot N \cdot L_{\mathrm{UAV}-i},
$$

where $L_{\mathrm{UAV}-i}$ is the average losses between the aerial relay node and a ground user $i$, and $N$ is the noise power.

We aim to find an efficient placement of the aerial relay node and the power allocation such that the total throughput of ground users is maximized. The optimization problem is given by:

$$
\underset{x_{u}, y_{u}, z_{u}, P}{\operatorname{maximize}} \sum_{i=1}^{M} B_{\mathrm{UAV}-i} \cdot \log _{2}\left(1+\mathrm{SNR}_{\mathrm{UAV}-i}\right)
$$

subject to $\sum_{i=1}^{M} B_{\mathrm{UAV}-i} \cdot \log _{2}\left(1+S \mathrm{NR}_{\mathrm{UAV}-i}\right) \leq B_{B S} \cdot \log _{2}\left(1+\mathrm{SNR}_{\mathrm{BS}-\mathrm{UAV}}\right)$,

$$
\begin{gathered}
\mathrm{SNR}_{\mathrm{UAV}-i} \geq \mathrm{SNR}_{\mathrm{th}}, \forall i \in M, \\
\sum_{i=1}^{M} p_{i} \leq p^{\max }, \\
p_{i} \geq 0, \forall i \in M, \\
x_{\min } \leq X_{u} \leq x_{\max }, \\
y_{\min } \leq Y_{u} \leq y_{\max }, \\
z_{\min } \leq Z_{u} \leq z_{\max } .
\end{gathered}
$$

Here, the first constraint set ensures that the data rate of the link that connected the aerial relay node with a ground user is less than or equal to the data rate of the link that connected the ground base station with the aerial relay node. The second constraint set guarantees that the signal-to-noise ratio of each ground user $i \in M$ is greater or equal to the threshold value $\mathrm{SNR}_{\mathrm{th}}$. The third constraint set ensures that the total power consumed by the aerial relay node must not be greater than its maximum power level $p^{\max }$. The fourth constraint set guarantees that the values of the power consumed by the aerial relay node take values greater than or equal to zero. The remaining constraints represent the maximum and allowed minimum values for $X_{u}, Y_{u}$, and $Z_{u}$. 


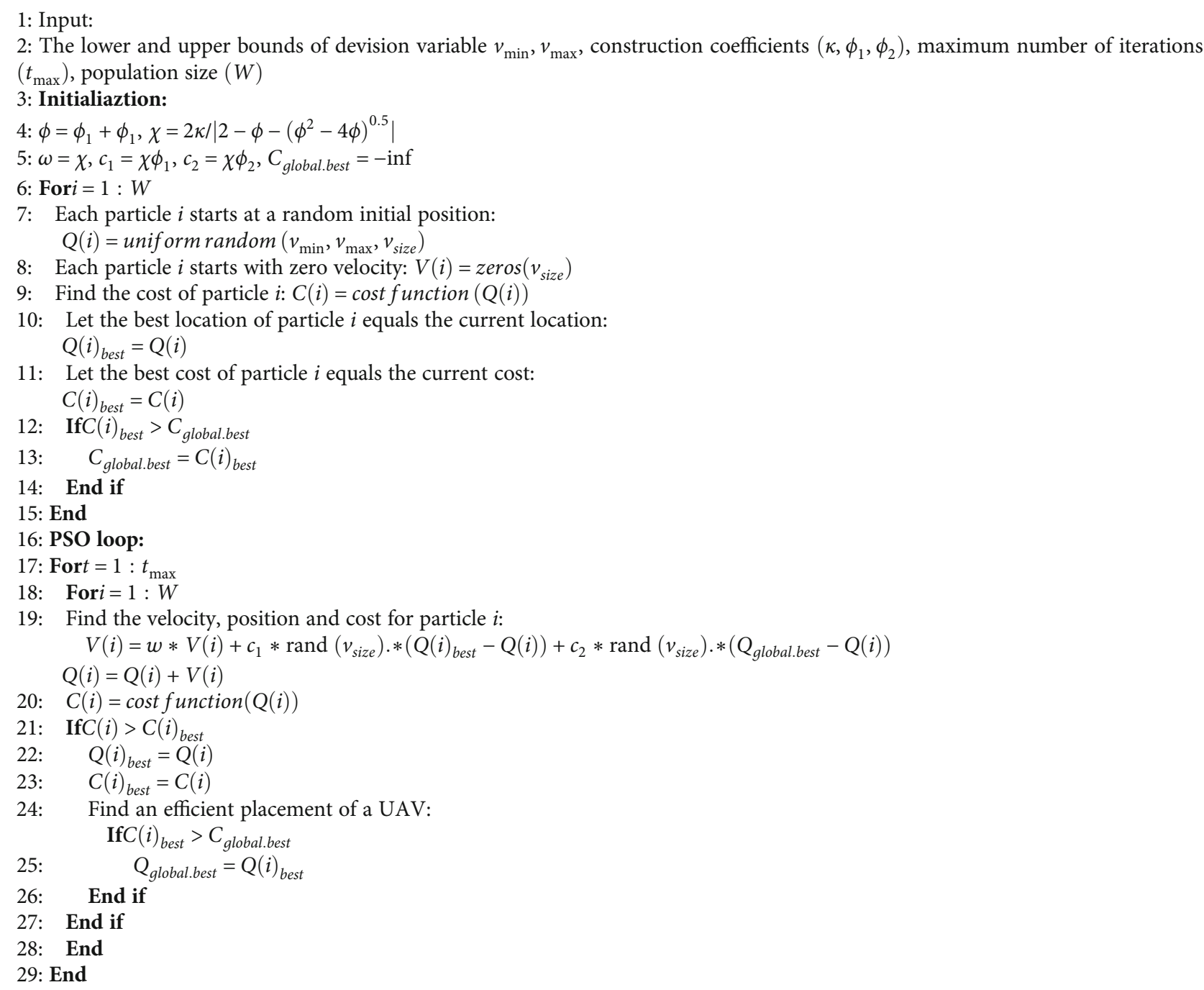

Algorithm 1: Efficient Aerial Relay Node Placement using PSO Algorithm.

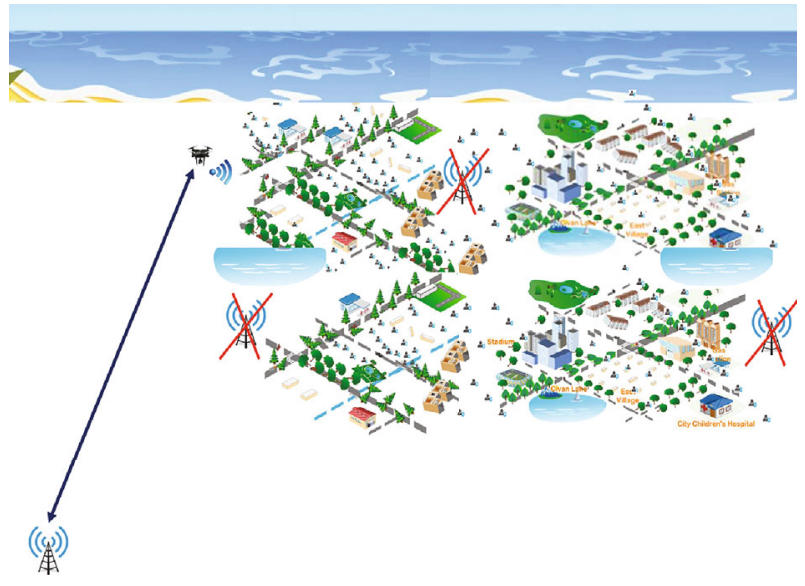

FIgURE 4: Scenario description: the disaster affected subarea, $\mathbb{R}$.
Finding the optimal 3D placement of the aerial relay node and the power allocation is generally difficult because the optimization problem is nonconvex. Therefore, in the next section, to find an efficient solution for the optimization problem, we present the particle swarm optimization algorithm and water-filling algorithm.

\section{Efficient Placement of Aerial Relay Node}

The aerial relay node placement is first optimized using the particle swarm optimization algorithm [22,23], with the total path loss from the aerial relay node to users minimized since the formulated joint optimization problem is nonconvex. Then, using an efficient location of the aerial relay node, the power allocation using the water-filling algorithm [24] is optimized to maximize the network's sum rate.

First, we apply the particle swarm optimization algorithm at each 2D plane ( $y z$-plane) to find an efficient placement for the aerial relay node that minimizes the total path loss for ground users. Second, at each efficient placement, we apply 


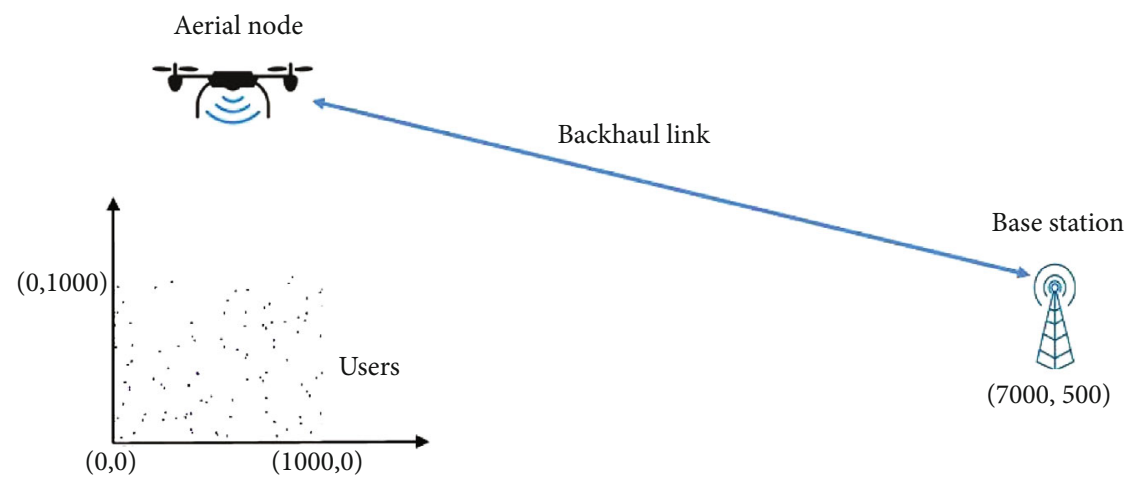

Figure 5: Users distribution inside affected subarea, $\mathbb{R}$, remote base station.

TABLE 1: System and simulation parameters.

\begin{tabular}{|c|c|c|c|c|c|}
\hline \multicolumn{3}{|c|}{ Simulation parameters } & \multicolumn{3}{|c|}{ System and algorithm parameters } \\
\hline Subarea $(\mathscr{R})$ dimensions & $\left(x_{1}, y_{1}\right),\left(x_{2}, y_{2}\right)$ & $(0,0),(1000 \mathrm{~m}, 1000 \mathrm{~m})$ & Carrier frequency & $f_{c}$ & $2 \mathrm{GHz}$ \\
\hline Number of ground users & M & 100 & Noise power & $\mathrm{Np}$ & $-120 \mathrm{dBm}$ \\
\hline Max. GBs transmit power & $P_{t_{\mathrm{GB}} \max }$ & $46 \mathrm{dBm}$ & Signal-to-noise threshold & SNR & $35 \mathrm{~dB}$ \\
\hline Max. UAV transmit power & $P_{t_{\mathrm{UAV}_{\max }}}$ & $30 \mathrm{dBm}$ & PSO population size & $N_{-p o p}$ & 50 \\
\hline Total UAV bandwidth & $B_{\mathrm{UAV}}$ & $50 \mathrm{MHz}$ & Max \# of iterations of PSO & $N_{\text {it }}$ & 50 \\
\hline Total GBs bandwidth & $B_{\mathrm{GBs}}$ & $100,75 \mathrm{MHz}$ & Environment parameters & $a, b$ & $9.6,0.28$ \\
\hline Base station location & $\left(x_{\mathrm{Bs}}, y_{\mathrm{Bs}}\right)$ & $(7000,500)$ & Environment parameters & $\eta_{L S O}, \eta_{\mathrm{NLSO}}$ & 1,20 \\
\hline
\end{tabular}

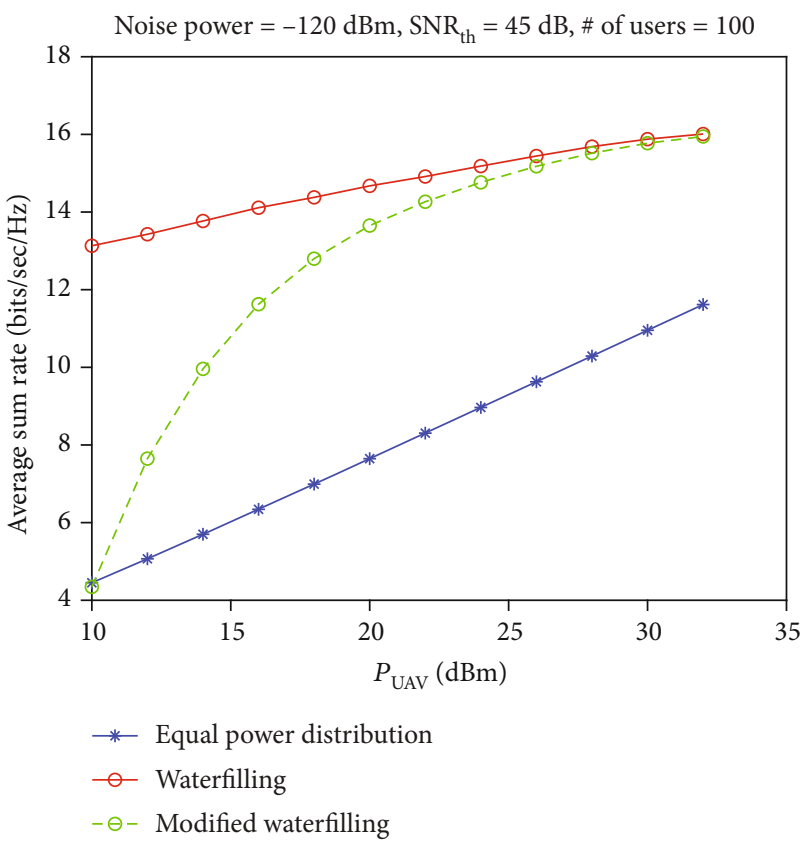

Figure 6: Average sum rate vs. total UAV transmission power.

the water-filling algorithm where each user must satisfy the $\mathrm{SNR}_{\text {th }}$ and maximize the data rate using the remaining power. Third, we calculate the data rate of the link that connected the ground base station with the aerial relay node ( $\left.B_{\mathrm{BS}} \cdot \log _{2}\left(1+\mathrm{SNR}_{\mathrm{BS}-\mathrm{UAV}}\right)\right)$ at each efficient placement. Finally, we find the maximum data rate of the link that con- nected the aerial relay node with the ground users $\left(\sum_{i=1}^{M}\right.$ $\left.B_{\mathrm{UAV}-i} \cdot \log _{2}\left(1+\mathrm{SNR}_{\mathrm{UAV}-i}\right)\right)$ that satisfies $\left(\sum_{i=1}^{M} B_{\mathrm{UAV}-i} \cdot \log _{2}(\right.$ $\left.\left.1+\mathrm{SNR}_{\mathrm{UAV}-i}\right) \leq B_{\mathrm{BS}} \cdot \log _{2}\left(1+\mathrm{SNR}_{\mathrm{BS}-\mathrm{UAV}}\right)\right)$.

The algorithm for particle swarm optimization, shown in Algorithm 1, begins with different candidates $(W)$ and attempts to develop candidate solutions iteratively based on each candidate's best global experience $\left(Q_{\text {global.best }}\right)$ and best experience $\left(Q(i)_{\text {best }}\right)$. The best position for each particle $(Q$ $\left.(i)_{\text {best }}\right)$ and the best global location $\left(Q_{\text {global.best }}\right)$ are modified in each step, and the particle velocities and positions are determined based on them [27]. The value of the velocity shows how often the position can be modified. This value is given by:

$$
\begin{aligned}
V(i)= & w * V(i)+c_{1} * \text { rand }\left(v_{\text {size }}\right) \cdot *\left(Q(i)_{\text {best }}-Q(i)\right) \\
& +c_{2} * \text { rand }\left(v_{\text {size }}\right) \cdot *\left(Q_{\text {global.best }}-Q(i)\right),
\end{aligned}
$$

where $w$ is the weight of inertia, the personal and global learning coefficients are $c_{1}$ and $c_{2}$ and rand $\left(v_{\text {size }}\right)$ are random positive numbers. Also, each particle's position is changed as:

$$
Q(i)=Q(i)+V(i)
$$

The time complexity of the PSO algorithm will depend on the number of iterations $\left(t_{\max }\right)$ and the number of candidate solutions $(W)$. The convergence speed was studied for the PSO algorithm [23]. This study has led to guidelines for choosing a set of coefficients $\left(\kappa, \phi_{1}, \phi_{2}\right)$ that are believed to 


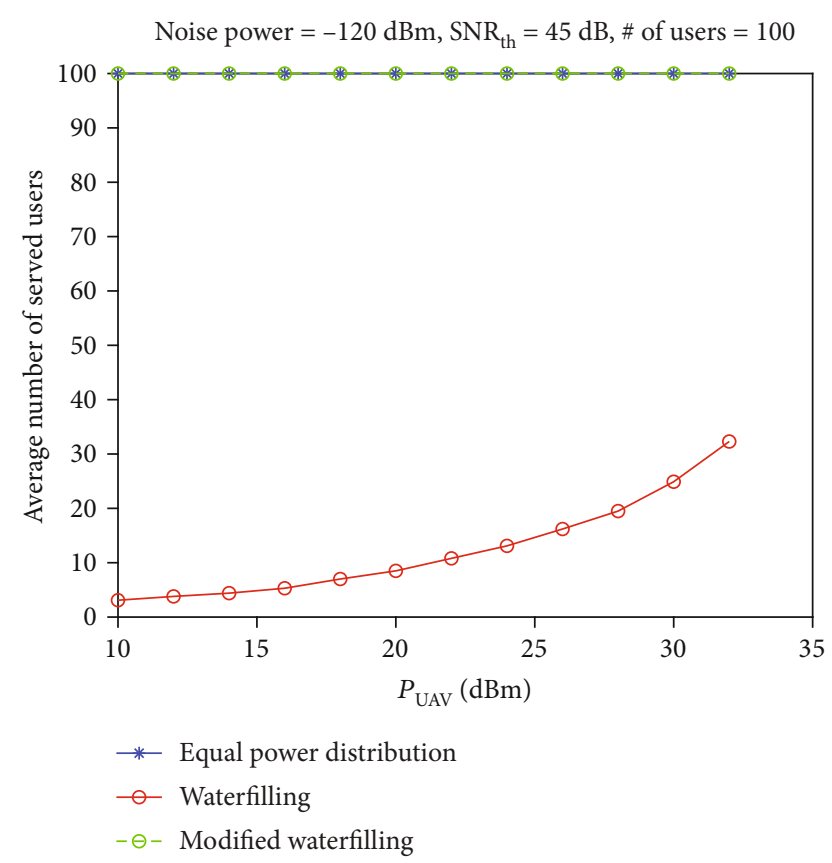

FIgURE 7: Average served users vs. total UAV transmission power.

induce convergence to a point and prevent the particles of the swarm from diverging. Based on this analysis, we selected our parameters (see Algorithm 1 (steps 4 and 5)).

\section{Simulation Results and Analysis}

Consider a coverage subarea denoted as $\mathbb{R}$ where the natural disaster happens. Figure 4 illustrates the affected area, $\mathbb{R}$. This work is aimed at using a single UAV as a relay node and aerial base station and finding its efficient placement such that the total throughput of ground users is maximized. More specifically, a single UAV is used as a relay node with a remote terrestrial base station and to provide wireless coverage for ground users in downlink scenarios during a disastrous situation. The wireless devices are nonuniformly distributed inside the targeted subarea using a nonuniform beta random distribution function $f(x, y)$. In this section, the optimization problem's solution is developed using three approaches; (1) the equal power distribution approach, (2) the water filling approach, and (3) the modified water filling approach. The simulation results are presented in these proposed approaches to enhance the total data rate and the number of served wireless devices.

In this work, the particle swarm optimization algorithm is first used to find an efficient 3D placement of the aerial relay node (the UAV), such that the total path loss of the ground users is minimized. Then, the equal power distribution, water filling, and modified water filling approaches are used to distribute the transmission power of the UAV $P_{\mathrm{UAV}}$ among the users. The water filling algorithm provides an optimal power allocation for frequency division multiple access subchannels [24], such that the throughput is maximized, where the power is divided between the users accord- ing to each user's path loss. Specifically, it assigns high power to the user who has the minimum path loss. While it gives zero power to users having path loss higher than the threshold, this threshold is determined according to the total transmission power.

In this work, we consider using a single UAV in a disaster situation, where the objective is to serve all ground users such that the sum data rate is maximized.

Consequently, a modified water-filling algorithm is utilized. In this algorithm, we first distribute part of the transmitted power among the users, satisfying that each user has an amount of power that makes the user signal-to-noise ratio $\mathrm{SNR}_{i}$ is equal to the $\mathrm{SNR}_{\mathrm{t}_{\mathrm{h}}}$. Then, the remaining transmitted power is distributed between the users according to the water-filling algorithm. For benchmarking and performance evaluation, the equal power allocation method is performed. Then, this method will be compared with water filling and modified water-filling algorithms.

In this scenario, the UAV is used as a relay node immediately after the disaster occurred, where the terrestrial base stations are disrupted and went out of service in the disaster regions, as shown in Figure 4. The contingency plan is to restore network coverage as quickly as possible to help both residents of the affected area and the rescue team members during the search and rescue operations to communicate with the authorities to meet their needs. UAV can be used as a relay node between the ground users and the nearest working base station. Here, we consider a square geographical area with minimum and maximum points of $\left(x_{1}, y_{1}\right)$ and $\left(x_{2}, y_{2}\right)$, as a coverage region where the natural disaster occurs. Moreover, the nearest base station is located at point $\left(x_{B S}, y_{B s}\right)$, as shown in Figure 5. The ground users are nonuniformly distributed inside the subarea with dimensions $1000 \mathrm{~m} \times 1000 \mathrm{~m}$ as depicted in Figure 5. First, the UAV aerial node's efficient location is evaluated by particle swarm optimization algorithm, such that the average throughput of the ground users is enhanced. Table 1 presents the parameters used in this simulation.

Figure 6 shows the performance of the three algorithms that are considered in this work in terms of average sum rate versus the UAV transmission power $P_{\mathrm{UAV}}$ at the UAV efficient placement. As expected, the water filling algorithm outperforms the other two algorithms. We also notice that the modified water filling algorithm performance improves as the transmission power increases until it reaches a performance asymptotic to the water filling algorithm at transmission power greater than $30 \mathrm{dBm}$. The reason for that, the modified water filling algorithm distributes the transmission power in two stages. The first stage is to fulfill the constraints in Eq. (6a) by assigning power to whole users to satisfy the condition $\mathrm{SNR}_{i} \geq S N R_{t h}$. The second stage uses a waterfilling algorithm to distribute the remaining transmission power among the users. Since a part of the transmission power is used to satisfy the condition, $\mathrm{SNR}_{i}=\mathrm{SNR}_{\mathrm{th}}$; hence, as we increase the transmission power $P_{\mathrm{UAV}}$, the second stage will have more power to use for the water-filling power algorithm. At high $P_{\mathrm{UAV}}$, the percentage of power used to satisfy the condition $\mathrm{SNR}_{i}=\mathrm{SNR}_{\mathrm{th}}$ will become negligible. Hence, 


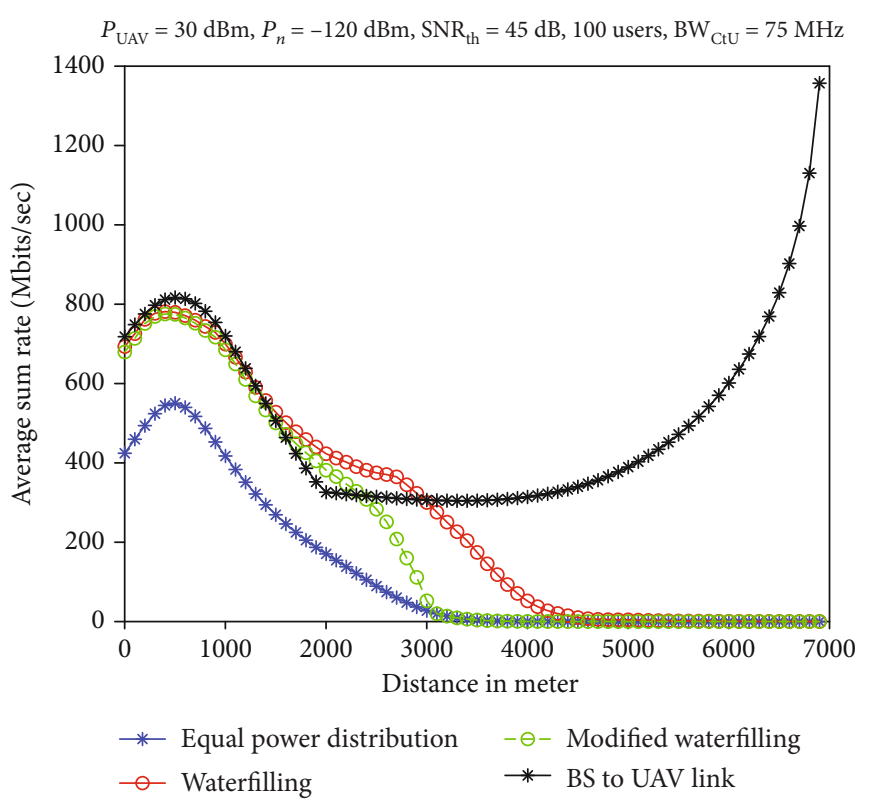

(a) Average sum rate vs. BS power at $75 \mathrm{MHz}$ bandwidth

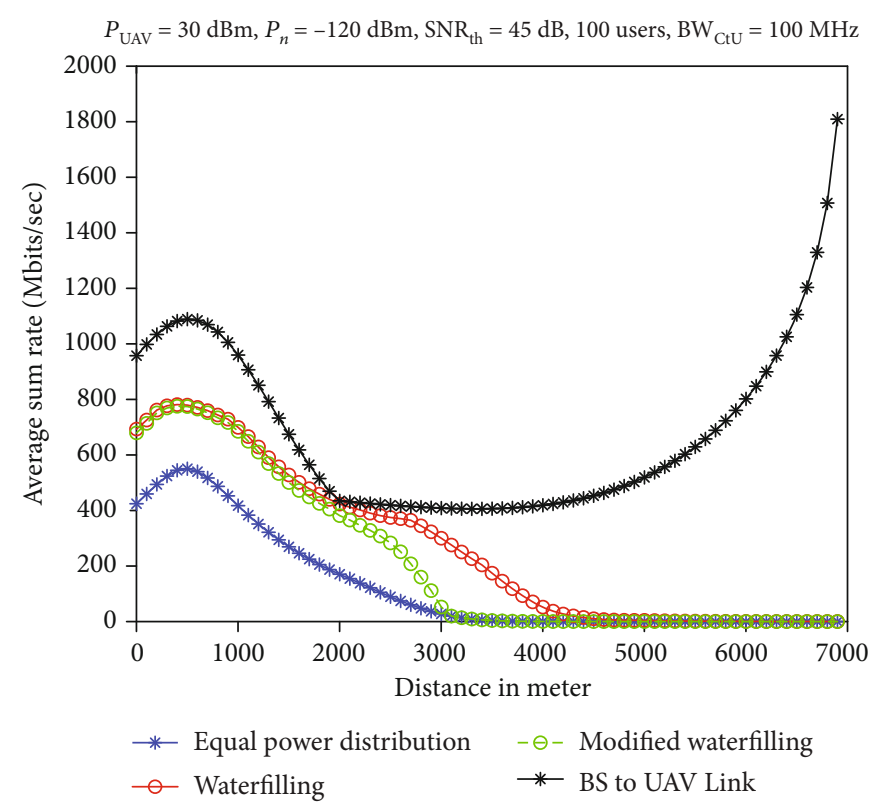

(b) Average sum rate vs. BS power at $100 \mathrm{MHz}$ bandwidth

FIGURE 8: Average sum rate vs. ground base station power at 75 and $100 \mathrm{MHz}$ bandwidth.

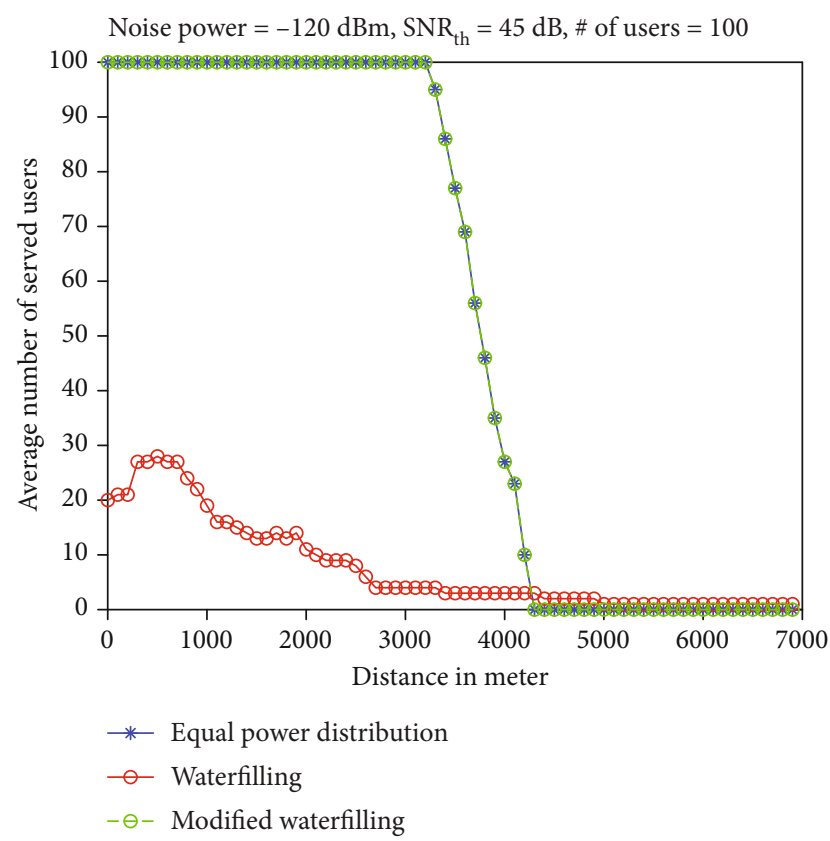

Figure 9: Average served users vs. distance (m).

the modified water-filling algorithm will have a performance that is comparable to the water-filling algorithm.

Since we assume the disaster scenario, the aim is to maximize the sum data rate and serve the largest possible users. Figure 6 shows the modified water-filling algorithm's performance compared with water filling and equal power algorithms at UAV efficient placement. In this scenario, we assumed that the total transmission bandwidth is $50 \mathrm{MHz}$, and we considered that the served user is the user who gets more than 50kbits/s. We depict that the whole users are served in both modified water-filling and equal power distribution algorithms. While, in the standard water-filling algorithm, the number of served users increased gradually as the transmission power increases. Specifically, at $P_{\mathrm{UAV}}=30$ $\mathrm{dBm}$, about $26 \%$ of the total users are only served. If we compare the performance of the considered algorithms in Figures 6 and 7, we conclude that the modified water filling is more appropriate for disaster situations.

In this scenario, we consider that 100 wireless devices are nonuniformly distributed inside a square area with $1000 \times$ 1000 meters in $x y$ plane, and the base station is located at the point $(7000,500,30)$ in the $x y z$ space, as shown in Figure 5. We evaluate the aerial node's performance for the three algorithms of power distribution that considered in this work along the $x$-axis at efficient locations of the $y z$ axis, at $P_{\mathrm{UAV}}=30 \mathrm{dBm}$, as shown in Figure 8. Moreover, we evaluate the sum rate of the backhaul link between the aerial node and the base station at two different bandwidths $\mathrm{BW}=75$ and $100 \mathrm{MHz}$. Figure 8 also shows that the water-filling algorithm outperforms the other two algorithms along with the horizontal distance ( $x$-axis). The backhaul link always has a data rate greater than the three power distribution algorithms at the efficient location $(500,502.3,271.9)$. It is observed that the average sum rate for the backhaul link is $1089 \mathrm{Mbits} / \mathrm{sec}$. Moreover, it is shown that the average sum rate for the modified water-filling algorithm is $774.4 \mathrm{Mbits} / \mathrm{sec}$ with ensuring $100 \%$ user coverage. In contrast, the average sum rate for the water filling is $779.5 \mathrm{Mbits} / \mathrm{sec}$ with only $28 \%$ user coverage. Therefore, at backhaul bandwidth $100 \mathrm{MHz}$, the backhaul link always fulfilled the constraint of Eq. (6a), that the backhaul link has a greater data rate than whole power distribution algorithms.

Figure 9 shows the three power distribution algorithms' performance in terms of the number of served users along 
TABLE 2: Simulation results for the proposed algorithms within a subarea $\mathbb{R}$.

\begin{tabular}{|c|c|c|c|c|c|c|c|}
\hline Step & Algorithm & $\begin{array}{c}\text { Efficient UAV } \\
\text { placement }\end{array}$ & $\begin{array}{l}\text { Total path loss UAV } \\
\text { to users }(\mathrm{dB})\end{array}$ & $\begin{array}{l}\text { GBs-to-UAV backhaul } \\
\text { path loss }(\mathrm{dB})\end{array}$ & $\begin{array}{c}\text { Average sum rate } \\
\text { (Mbits/sec) }\end{array}$ & $\begin{array}{c}\text { CtU average rate } \\
\text { (Mbits/sec) }\end{array}$ & $\begin{array}{l}\text { Num. of } \\
\text { served users }\end{array}$ \\
\hline 1 & $\begin{array}{c}\text { Eq. power } \\
\text { distr. } \\
\text { Water- } \\
\text { filling } \\
\text { Mod. } \\
\text { Water- } \\
\text { Filling }\end{array}$ & $(0,498.4,442)$ & 3751.18 & 102.99 & $\begin{array}{l}424.3 \\
693.2 \\
693.2\end{array}$ & 957.1 & $\begin{array}{c}100 \\
20 \\
100\end{array}$ \\
\hline 2 & $\begin{array}{l}\text { Eq. power } \\
\text { distr. } \\
\text { Water- } \\
\text { filling } \\
\text { Mod. } \\
\text { water- } \\
\text { filling }\end{array}$ & $\begin{array}{c}(500,502.3 \\
271.9)\end{array}$ & 3349.29 & 99.061 & $\begin{array}{l}550.4 \\
779.5 \\
774.4\end{array}$ & 1089 & $\begin{array}{c}100 \\
28 \\
100\end{array}$ \\
\hline 3 & $\begin{array}{l}\text { Eq. power } \\
\text { distr. } \\
\text { Water- } \\
\text { filling } \\
\text { Mod. } \\
\text { water- } \\
\text { filling }\end{array}$ & $\begin{array}{c}(1000,494.8 \\
429.5)\end{array}$ & 3727.80 & 106.22 & $\begin{array}{c}417.5 \\
699.3 \\
685\end{array}$ & 959.8 & $\begin{array}{c}100 \\
19 \\
100\end{array}$ \\
\hline 4 & $\begin{array}{l}\text { Eq. power } \\
\text { distr. } \\
\text { Water- } \\
\text { filling } \\
\text { Mod. } \\
\text { water- } \\
\text { filling }\end{array}$ & $\begin{array}{c}(1500,499.1 \\
706.6)\end{array}$ & 4183.68 & 115.25 & $\begin{array}{l}269.2 \\
527.9 \\
500.7\end{array}$ & 674.5 & $\begin{array}{c}100 \\
13 \\
100\end{array}$ \\
\hline 5 & $\begin{array}{l}\text { Eq. power } \\
\text { distr. } \\
\text { Water- } \\
\text { filling } \\
\text { Mod. } \\
\text { water- } \\
\text { filling }\end{array}$ & $\begin{array}{c}(2000,491.7 \\
997.2)\end{array}$ & 4503.21 & 122.67 & $\begin{array}{c}170.5 \\
423 \\
381.6\end{array}$ & 434.7 & $\begin{array}{c}100 \\
11 \\
100\end{array}$ \\
\hline 6 & $\begin{array}{l}\text { Eq. power } \\
\text { distr. } \\
\text { Water- } \\
\text { filling } \\
\text { Mod. } \\
\text { water- } \\
\text { filling }\end{array}$ & $\begin{array}{c}(3000,493.5 \\
1000)\end{array}$ & 5281.48 & 123.0 & $\begin{array}{c}27.52 \\
300.1 \\
52.2\end{array}$ & 408.6 & $\begin{array}{c}100 \\
4 \\
100\end{array}$ \\
\hline 7 & $\begin{array}{c}\text { Eq. power } \\
\text { distr. } \\
\text { Water- } \\
\text { filling } \\
\text { Mod. } \\
\text { water- } \\
\text { filling }\end{array}$ & $\begin{array}{l}(4000,500 \\
1000)\end{array}$ & 6227.37 & 123.90 & $\begin{array}{c}0.41 \\
52.12 \\
0.41\end{array}$ & 418.8 & $\begin{array}{c}27 \\
3 \\
27\end{array}$ \\
\hline 8 & $\begin{array}{c}\text { Eq. power } \\
\text { distr. } \\
\text { Water- } \\
\text { filling } \\
\text { Mod. } \\
\text { water- } \\
\text { filling }\end{array}$ & $\begin{array}{l}(5000,500, \\
1000)\end{array}$ & 6801.04 & 125.56 & $\begin{array}{l}0.02 \\
4.48 \\
0.02\end{array}$ & 519.2 & $\begin{array}{l}0 \\
2 \\
0\end{array}$ \\
\hline 9 & & & 7123.16 & 124.89 & & 801.9 & \\
\hline
\end{tabular}


TABLE 2: Continued.

\begin{tabular}{|c|c|c|c|c|c|c|c|}
\hline Step & Algorithm & $\begin{array}{c}\text { Efficient UAV } \\
\text { placement }\end{array}$ & $\begin{array}{l}\text { Total path loss UAV } \\
\text { to users }(\mathrm{dB})\end{array}$ & $\begin{array}{l}\text { GBs-to-UAV backhaul } \\
\text { path loss }(\mathrm{dB})\end{array}$ & $\begin{array}{c}\text { Average sum rate } \\
\text { (Mbits/sec) }\end{array}$ & $\begin{array}{c}\text { CtU average rate } \\
\text { (Mbits/sec) }\end{array}$ & $\begin{array}{l}\text { Num. of } \\
\text { served users }\end{array}$ \\
\hline & $\begin{array}{l}\text { Eq. power } \\
\text { distr. } \\
\text { Water- } \\
\text { filling } \\
\text { Mod. } \\
\text { water- } \\
\text { filling }\end{array}$ & $\begin{array}{c}(6000,499 \\
1000)\end{array}$ & & & $\begin{array}{c}0.004 \\
0.74 \\
0.004\end{array}$ & & $\begin{array}{l}0 \\
1 \\
0\end{array}$ \\
\hline 10 & $\begin{array}{c}\text { Eq. power } \\
\text { distr. } \\
\text { Water- } \\
\text { filling } \\
\text { Mod. } \\
\text { water- } \\
\text { filling }\end{array}$ & $\begin{array}{c}(7000,496.8 \\
1000)\end{array}$ & 7317.82 & 117.35 & $\begin{array}{c}0.0016 \\
0.26 \\
0.0016\end{array}$ & 1809 & $\begin{array}{l}0 \\
1 \\
0\end{array}$ \\
\hline
\end{tabular}

the $x$-axis, at $P_{\text {uav }}=30 \mathrm{dBm}$. It is clear from this figure that the modified water-filling and the equal power distribution algorithms always serve whole users along with the horizontal distance ( $x$-axis) from 0 to 4000 meters. The location of the aerial node in the $y z$ plane is evaluated using the particle swarm algorithm. In contrast, the water-filling algorithm can only serve $28 \%$ of whole users in the best-case scenario. This is because the power allocated by the water-filling algorithm is proportional to the users' channel quality. Specifically, the users with a better channel get more power, and the user with bad channels get less or no power.

Table 2 shows the efficient $(y, z)$ placement of the relay aerial base station and the corresponding minimum total path loss required to cover all ground users. It also shows the path loss between the ground base station and the aerial relay node at different horizontal locations $(x=1$ to 7000 $\mathrm{m}$ ) using particle swarm optimization algorithm for the three proposed algorithms; equal power allocation, water filling, and modified water filling. Moreover, this table presents the provided average sum rate from the relay node to the users and the total number of served wireless devices for each algorithm. It can be seen that from step number $6(x=3000 \mathrm{~m})$, the efficient altitude of the relay node is the maximum allowed altitude $z_{\max }=1000$ for this scenario. Therefore, as the relay node moves far from the coverage region, the distance between the relay node and ground station increased; consequently, the total path loss will be increased. Additionally, the average sum rate and the number of served users will be greatly decreased due to the considerable distance between the aerial node and the coverage region.

The modified water-filling algorithm is more feasible in disaster scenarios since a communication network should be provided to most users in the affected area to give them the ability to request assistance in case of need. In fact, in modified water-filling algorithm, we accomplished two goals; first, we ensure that all users are served even at low $P_{\text {UAVmax }}$, and second, the amount of transmitted power increases, and the UAV cell's overall performance improves.

\section{Conclusions}

Due to the large distance between the ground base station and the users located inside the coverage region where the natural disaster happened, a UAV was used as an aerial relay node to provide wireless coverage for wireless devices. In this work, a cellular-to-UAV path loss model was utilized for the backhaul connection between the ground base station and the UAV. Moreover, the air-to-ground path loss model was utilized for a downlink connection between the UAV and the ground users. Then, the trade-off introduced by these models was discussed. Specifically, the problem of finding an efficient placement of a UAV was formulated as an optimization problem, where the objective is to maximize wireless devices' total throughput. The particle swarm optimization and the modified water-filling algorithm were used to find an efficient UAV placement that maximizes the overall throughput. As future work, we propose to study the uplink scenario utilizing the ground-to-air path loss model. Moreover, we propose to extend this work using mm-wave bands, which provide very high bandwidth.

\section{Data Availability}

Data are available on request.

\section{Conflicts of Interest}

The authors declare that they have no conflicts of interest.

\section{Acknowledgments}

The authors extend their appreciation to the Deputyship for Research and Innovation, Ministry of Education - Kingdom of Saudi Arabi for funding this research work through the project number-68642020 IF. 


\section{References}

[1] A. Al-Hourani, S. Kandeepan, and A. Jamalipour, "Modeling air-to-ground path loss for low altitude platforms in urban environments," in 2014 IEEE global communications conference, pp. 2898-2904, Austin, TX, USA, 2014.

[2] M. Mozaffari, W. Saad, M. Bennis, and M. Debbah, "Drone small cells in the clouds: design, deployment and performance analysis," in 2015 IEEE global communications conference (GLOBECOM), pp. 1-6, San Diego, CA, USA, 2015.

[3] A. Sawalmeh, N. S. Othman, H. Shakhatreh, and A. Khreishah, "Providing wireless coverage in massively crowded events using uavs," in 2017 IEEE 13th Malaysia International Conference on Communications (MICC), pp. 158-163, Johor Bahru, Malaysia, 2017.

[4] H. Shakhatreh, A. H. Sawalmeh, A. Al-Fuqaha et al., "Unmanned aerial vehicles (uavs): a survey on civil applications and key research challenges," IEEE Access, vol. 7, pp. 48572-48634, 2019.

[5] A. H. Sawalmeh, N. S. Othman, H. Shakhatreh, and A. Khreishah, "Wireless coverage for mobile users in dynamic environments using uav," IEEE Access, vol. 7, pp. 126376126390, 2019.

[6] S. Yin, Z. Qu, and L. Li, "Uplink resource allocation in cellular networks with energy-constrained uav relay," in 2018 IEEE 87th Vehicular Technology Conference (VTC Spring), pp. 1-5, Porto, Portugal, 2018.

[7] U. Demir, C. Toker, and O. Ekici, "Energy-efficient deployment of uav in v2x network considering latency and backhaul issues," in 2020 IEEE International Black Sea Conference on Communications and Networking (BlackSeaCom), pp. 1-6, Odessa, Ukraine, 2020.

[8] S. Kumar, S. Suman, and S. De, "Backhaul and delay-aware placement of uav-enabled base station," in IEEE INFOCOM 2018-IEEE Conference on Computer Communications Workshops (INFOCOM WKSHPS), pp. 634-639, Honolulu, HI, USA, 2018.

[9] Y. Chen, W. Feng, and G. Zheng, "Optimum placement of uav as relays," IEEE Communications Letters, vol. 22, no. 2, pp. 248-251, 2017.

[10] P. Zhan, K. Yu, and A. L. Swindlehurst, "Wireless relay communications with unmanned aerial vehicles: performance and optimization," IEEE Transactions on Aerospace and Electronic Systems, vol. 47, no. 3, pp. 2068-2085, 2011.

[11] Y. He, D. Zhai, D. Wang, X. Tang, and R. Zhang, "A relay selection protocol for uav-assisted vanets," Applied Sciences, vol. 10, no. 23, article 8762, 2020.

[12] T. D. P. Perera, D. N. K. Jayakody, S. Garg, N. Kumar, and L. Cheng, "Wireless-powered uav assisted communication system in Nakagami-m fading channels," in 2020 IEEE 17th Annual Consumer Communications \& Networking Conference (CCNC), pp. 1-6, Las Vegas, NV, USA, 2020.

[13] Y. Zeng, Q. Wu, and R. Zhang, "Accessing from the sky: a tutorial on uav communications for $5 \mathrm{~g}$ and beyond," Proceedings of the IEEE, vol. 107, no. 12, pp. 2327-2375, 2019.

[14] L. Zhang, H. Zhao, S. Hou et al., "A survey on 5 g millimeter wave communications for uav-assisted wireless networks," IEEE Access, vol. 7, pp. 117460-117504, 2019.

[15] H. Ullah, N. G. Nair, A. Moore, C. Nugent, P. Muschamp, and M. Cuevas, " $5 \mathrm{~g}$ communication: an overview of vehicle-toeverything, drones, and healthcare use-cases," IEEE Access, vol. 7, pp. 37251-37268, 2019.
[16] C. Yan, L. Fu, J. Zhang, and J. Wang, "A comprehensive survey on uav communication channel modeling," IEEE Access, vol. 7, pp. 107769-107792, 2019.

[17] H. Hildmann and E. Kovacs, "Using unmanned aerial vehicles (uavs) as mobile sensing platforms (msps) for disaster response, civil security and public safety," Drones, vol. 3, no. 3, p. 59, 2019.

[18] S. Shakoor, Z. Kaleem, M. I. Baig, O. Chughtai, T. Q. Duong, and L. D. Nguyen, "Role of uavs in public safety communications: energy efficiency perspective," IEEE Access, vol. 7, pp. 140665-140679, 2019.

[19] Z. Ullah, F. Al-Turjman, and L. Mostarda, "Cognition in uavaided $5 \mathrm{~g}$ and beyond communications: a survey," IEEE Transactions on Cognitive Communications and Networking, vol. 6, no. 3, pp. 872-891, 2020.

[20] A. Al-Hourani and K. Gomez, "Modeling cellular-to-uav pathloss for suburban environments," IEEE Wireless Communications Letters, vol. 7, no. 1, pp. 82-85, 2017.

[21] A. Al-Hourani, S. Kandeepan, and S. Lardner, "Optimal lap altitude for maximum coverage," IEEE Wireless Communications Letters, vol. 3, no. 6, pp. 569-572, 2014.

[22] J. Kennedy and R. Eberhart, "Particle swarm optimization," in Proceedings of ICNN'95-International Conference on Neural Networks, vol. 4, pp. 1942-1948, Perth, WA, Australia, 1995.

[23] M. Clerc and J. Kennedy, "The particle swarm - explosion, stability, and convergence in a multidimensional complex space," IEEE Transactions on Evolutionary Computation, vol. 6, no. 1, pp. 58-73, 2002.

[24] D. P. Palomar and J. R. Fonollosa, "Practical algorithms for a family of waterfilling solutions," IEEE Transactions on Signal Processing, vol. 53, no. 2, pp. 686-695, 2005.

[25] S. I. Alnagar, A. M. Salhab, and S. A. Zummo, "Unmanned aerial vehicle relay system: performance evaluation and $3 \mathrm{~d}$ location optimization," IEEE Access, vol. 8, pp. 67635-67645, 2020.

[26] A. A. Khuwaja, Y. Chen, N. Zhao, M.-S. Alouini, and P. Dobbins, "A survey of channel modeling for uav communications," IEEE Communications Surveys \& Tutorials, vol. 20, no. 4, pp. 2804-2821, 2018.

[27] E. Kalantari, H. Yanikomeroglu, and A. Yongacoglu, "On the number and $3 \mathrm{~d}$ placement of drone base stations in wireless cellular networks," in 2016 IEEE 84th Vehicular Technology Conference (VTC-Fall), pp. 1-6, Montreal, QC, Canada, 2016. 